\title{
The solution of a one-phase Stefan problem with a forcing term by homotopy analysis method
}

\author{
O. N. Onyejekwe \\ Department of Mathematics, Eastern Florida State College, USA \\ E-mail: onyejekweo@easternflorida.edu
}

Copyright $\odot 2014$ O.N.Onyejekwe. This is an open access article distributed under the Creative Commons Attribution License, which permits unrestricted use, distribution, and reproduction in any medium, provided the original work is properly cited.

\begin{abstract}
In this paper, we use the homotopy analysis method (HAM), to obtain the solutions of the temperature distribution, the position of the moving boundary and the Stefan condition. There are advantages to using HAM, firstly it is independent of small/large physical parameters, there is flexibility on the choice of base function and initial guess of solution and lastly there is great generality. The results obtained from this method shows high accuracy, computational efficiency and a strong rate of convergence.
\end{abstract}

Keywords: Dirichlet; Forcing Term; Homotopy Analysis Method; Stefan Problem.

\section{Introduction}

Many problems involving melting, freezing and evaporation of substances are characterized by a moving interface which changes from solid to liquid, from solid to gas, or vice versa respectively. These problems are known as moving boundary problems, free boundary problems or Stefan problems. A common characteristic to these problems is that the material in question undergoes a phase change accompanied by a moving boundary that has to be determined. The unknown boundary introduces a nonlinear form of the energy balance at the interface; as a result, analytical solutions are difficult to obtain except in some cases. Closed form solutions, though sometimes limited in scope provide benchmarks for testing and validating numerical procedures. With the advent of computers and sophisticated solution methods, numerical and approximate analytical techniques are now employed to solve different types of Stefan problems. Some of these methods include finite difference method[16]-[17], green element method[6], variable space grid[9]-[10],boundary immobilization schemes[8]-[10], adomain decomposition method[6]-[7], heat balance integral method[12],homotopy perturbation method[3], variation iteration method[5] and variable space grid [15].

In paper [4], Jafari et al used HAM to solve the Stefan problem with Dirichlet boundary conditions and no forcing term. In this paper, homotopy analysis method (HAM) proposed by Liao [11]-[13] and modified by Alomari [1] is used to solve a one-phase Stefan problem with a forcing term. The homotopy analysis method has been used in recent years to solve ordinary and partial differential equations [1]. Unlike the methods mentioned above, HAM avoids discretization, provides us with efficient numerical solution with high accuracy; minimal calculation and the avoidance of physical unrealistic assumptions. The convergence region for the series solution obtained by HAM is determined by the convergence-control parameter $\mathrm{h}$. We have considered two problems with analytical solutions, in order to make comparisons of numerical results with exact ones.

\section{Statement of the problem}

The temperature $u(x, t)$ satisfies the following heat conduction equation

$\frac{\partial u}{\partial t}-\frac{\partial^{2} u}{\partial x^{2}}=f(x, t), 0<x<s(t), t>0$

With the initial and boundary conditions respectively 
$u(x, 0)=g(x), \quad 0 \leq x \leq 1$,

$u\left(x^{*}, t\right)=z(t), t>0, x^{*} \in(0, s(t)), t>0$

$u(s(t), t)=0, t>0$,

Where $\mathrm{u}(\mathrm{x}, \mathrm{t})$ is the phase temperature, $\mathrm{s}(\mathrm{t})$ is the position of the moving boundary, $\mathrm{f}(\mathrm{x}, \mathrm{t}), \mathrm{g}(\mathrm{x})$ and $\mathrm{z}(\mathrm{t})$ are sufficiently smooth and nonnegative functions.

The moving interface s ( $\mathrm{t}$ ) satisfies the energy balance equation known as the Stefan condition

$\frac{d s}{d t}=-\frac{\partial u}{d x}, x=s(t), t>0$,

Subject to

$\mathrm{s}(\mathrm{t})=\mathrm{c}, \mathrm{t}=0$

Where $\mathrm{c}$ is any constant.

In paper [2], it is shown that Eqs. (2.1)- (2.5) has a unique solution.

\section{Homotopy analysis method}

To illustrate the basic idea of the HAM, we consider the following differential equation:

$N[u(x, t)]=k(x, t)$,

Where $\mathrm{N}$ is a nonlinear operator, $\mathrm{x}$ and $\mathrm{t}$ denotes independent and dependent variables respectively, $\mathrm{u}$ is an unknown function and $\mathrm{k}(\mathrm{x}, \mathrm{t})$ is the nonhomogeneous term. By means of HAM, we first construct a zeroth-order deformation equation

$(1-q) L\left[\varphi(x, t ; q)-u_{0}(x, t)\right]=q h N[\varphi(x, t ; q)-k(x, t)]$,

Where $q \in[0,1]$ is the embedding parameter, $h \neq 0$ is an convergence-control parameter, L is an auxiliary linear operator, $\varphi(x, t ; q)$ is an unknown factor, $u_{0}(x, t)$ is an initial guess of $\mathrm{u}(\mathrm{x}, \mathrm{t})$. It is obvious that when the embedding parameter $\mathrm{q}$ goes from 0 to 1 , the values for $\varphi(x, t ; q)$ becomes

$\varphi(x, t ; 0)=u_{0}(x, t), \quad \varphi(x, t ; 1)=u(x, t)$,

Respectively. Thus as $\mathrm{q}$ increases from 0 to 1 , the solution $\varphi(x, t ; q)$ varies from the initial guess $u_{0}(x, t)$ to the solution $\mathrm{u}(\mathrm{x}, \mathrm{t})$. Expanding $\varphi(x, t ; q)$ in Taylor series with respect to $\mathrm{q}$, we obtain

$\varphi(x, t ; q)=u_{0}(x, t)+\sum_{m=1}^{+\infty} u_{m}(x, t) q^{m}$,

Where

$u_{m}(x, t)=\left.\frac{1}{m !} \frac{\partial^{m} \varphi(x, t ; q)}{\partial q^{m}}\right|_{q=0}$,

The convergence of the series (3.4) depends upon the convergence-control parameter $h$.

With HAM, we have the freedom to choose the initial guess $u_{0}(x, t)$, the auxiliary linear operator L, and the nonzero convergence-control parameter $h$. We assume that all of them are properly chosen so that:

1) The solution $\varphi(x, t ; q)$ of the zeroth-order deformation equation (3.2) exists for all $q \in[0,1]$.

2) The homotopy analysis derivative $D_{m}(\varphi(x, t ; q))$ exists for $\mathrm{m}=1,2,3, \ldots,+\infty$.

3) The power series (3.4) of $\varphi(x, t ; q)$ converges at $\mathrm{q}=1$.

Then from Eqs (3.3) and (3.4), we have under these assumptions the solution series

$u(x, t)=u_{0}(x, t)+\sum_{m=1}^{+\infty} u_{m}(x, t)$,

Which must be one of the solutions of the original nonlinear equation, as proven by Liao [10] Define the vectors

$\overrightarrow{u_{n}}=\left\{u_{0}(x, t), u_{1}(x, t), \ldots, u_{n}(x, t)\right\}$,

Differentiating the zeroth-order deformation equation (3.2) $\mathrm{m}$ - times with respect to $\mathrm{q}$ and then dividing them by $\mathrm{m}$ ! And finally setting $\mathrm{q}=0$, we have the $\mathrm{mth}$ - order deformation equation

$L\left[u_{m}(x, t)-\chi_{m} u_{m-1}(x, t)\right]=h R_{m}\left(\begin{array}{c}\rightarrow \\ u_{m-1}\end{array}\right)$,

Where 
$R_{m}\left(\begin{array}{c}\rightarrow \\ u_{m-1}\end{array}\right)=\left.\frac{1}{(m-1) !} \frac{\partial^{m-1} N[\varphi(x, t ; q)-g(x, t)]}{\partial q^{m-1}}\right|_{q=0}$,

And

$\chi_{m}=\left\{\begin{array}{l}0, m \leq 1 \\ 1, m>1\end{array}\right.$,

It should be emphasized that $u_{m}(x, t)$ for $m \geq 1$ is governed by the linear equation (3.8) with linear boundary conditions that come from the original problem. If eq.(3.1) has a unique solution, then this method will produce a unique solution. If eq. (3.1) does not have a unique solution, then HAM, will give a solution among many other solutions.

\section{Method of solution}

In this paper, we use HAM to obtain the approximate analytical solution to the Stefan Problem by finding both the temperature $u(x, t)$ and the position of the moving boundary $\mathrm{s}(\mathrm{t})(2.1)-(2.5)$.

The following other conditions (2.2) and (2.3) are known.

Using the solution procedure by HAM, we define a linear operator in the form.

Solution procedure by HAM, we define a linear operator in the form

$L[\phi(x, t ; q)]=\frac{\partial \phi(x, t ; q)}{\partial t}$,

With the property

$L\left[c_{1}(x)\right]=0$,

Where $c_{1}(x)$ is the integration constant? The nonlinear operator is taking

$N[\phi(x, t ; q)]=-u_{t}(x, t ; q)+u_{x x}(x, t ; q)+f(x, t)$,

So we can define $R_{m}$ as

$R_{m}\left(\begin{array}{c}\rightarrow \\ u_{m-1}\end{array}\right)=-\frac{\partial u_{m-1}(x, t)}{\partial t}+\frac{\partial^{2} u_{m-1}(x, t)}{\partial x^{2}}+f(x, t)$

Using (3.6), (3.8) and (4.3), we can get

$u_{m}(x, t)=\chi_{m} u_{m-1}(x, t)+h \int R_{m}\left(\overrightarrow{u_{m-1}}\right) d t+c_{1}(x)$,

The parameters used during the application of HAM are as follows:

The initial guess is

$u_{0}(x, t)=u(x, 0)$,

The calculations for $u(x, t)$ and $s(t)$ are as follows:

$u(x, t)=\sum_{m=0}^{+\infty} u_{m}(x, t)=u_{0}+u_{1}+u_{2}+\ldots$

We set $\left(u\left(x^{*}, t\right)\right)_{H A M}=\left(u\left(x^{*}, t\right)\right)_{E X A C T}$ to solve for the convergence-control parameter $\mathrm{h}$ at each time step, and $(u(s(t), t))_{H A M}=(u(s(t), t))_{E X A C T}$, to solve for s (t) at each time step.

The approximate analytical solution for the Stefan condition is obtained by

$\left(\frac{d s}{d t}\right)_{H A M}=\left(-\frac{\partial u_{m-1}(x, t)}{\partial x}\right)_{H A M}=-\sum_{m=0}^{+\infty} u_{x m}(s(t), t)=-\left(u_{x 0}(s(t), t)+u_{x 1}(s(t), t)+u_{x 2}(s(t), t)+, \ldots\right)$

\section{Numerical examples}

Example 1: We consider Stefan Problem (2.1) - (2.5) with

$f(x, t)=x \exp (t)+2$

$g(x)=x(1-x)$.

$u\left(x^{*}, t\right)=\exp (t)-1, x^{*}=1$ 
Where the exact solution for $\mathrm{u}(\mathrm{x}, \mathrm{t})$ and $\mathrm{s}(\mathrm{t})$ respectively are $u(x, t)=x(\exp (t)-x), 0 \leq x \leq s(t)$

$s(t)=\exp (t), t \geq 0$.

The results obtained for $\mathrm{u}(\mathrm{x}, \mathrm{t})$ and $\mathrm{s}(\mathrm{t})$ and the Stefan condition, when $\mathrm{h}=1$ using HAM are listed below

Table 1: Results for $u(x, t)$ when $\mathrm{x}=0.25$

\begin{tabular}{|c|c|c|c|}
\hline $\mathrm{t}$ & Exact $\mathrm{u}(\mathrm{x}, \mathrm{t})$ & HAM & Absolute Error \\
\hline 0.0 & 0.1875 & 0.1875 & 0 \\
\hline 0.05 & 0.2003177740 & 0.2003177750 & $1.0 \times 10^{-9}$ \\
\hline 0.10 & 0.2137927295 & 0.2137927400 & $1.05 \times 10^{-8}$ \\
\hline 0.15 & 0.2279585608 & 0.2279585650 & $4.2 \times 10^{-9}$ \\
\hline 0.20 & 0.2428506895 & 0.2428507025 & $1.30 \times 10^{-8}$ \\
\hline 0.25 & 0.2585063542 & 0.2585063475 & $6.7 \times 10^{-9}$ \\
\hline 0.30 & 0.2749647020 & 0.2749647025 & $5.0 \times 10^{-10}$ \\
\hline 0.35 & 0.2922668872 & 0.2922668850 & $2.2 \times 10^{-9}$ \\
\hline 0.40 & 0.3104561745 & 0.3104561675 & $7.0 \times 10^{-9}$ \\
\hline 0.45 & 0.3295780462 & 0.3295780350 & $1.12 \times 10^{-8}$ \\
\hline 0.50 & 0.3496803178 & 0.3496803100 & $7.8 \times 10^{-8}$ \\
\hline 0.55 & 0.3708132545 & 0.3708132625 & $8.0 \times 10^{-9}$ \\
\hline 0.60 & 0.3930297000 & 0.3930297000 & 0 \\
\hline 0.65 & 0.4163852072 & 0.4163852150 & $7.8 \times 10^{-8}$ \\
\hline 0.70 & 0.4409381768 & 0.4409381875 & $1.07 \times 10^{-8}$ \\
\hline 0.75 & 0.4667500042 & 0.4667499975 & $6.7 \times 10^{-9}$ \\
\hline 0.80 & 0.4938852320 & 0.4938852225 & $9.5 \times 10^{-9}$ \\
\hline 0.85 & 0.5224117130 & 0.5224117075 & $5.5 \times 10^{-9}$ \\
\hline 0.90 & 0.5524007778 & 0.5524007750 & $2.8 \times 10^{-9}$ \\
\hline 0.95 & 1.715915505 & 1.715915503 & $5.2 \times 10^{-9}$ \\
\hline 1.0 & 1.841470985 & 1.841470985 & $9.5 \times 10^{-9}$ \\
\hline
\end{tabular}

Table 2: Results for s (t)

\begin{tabular}{cccc}
\hline $\mathrm{t}$ & Exact s(t) & HAM & Absolute Error \\
\hline 0.0 & 1 & 1 & 0 \\
0.05 & 1.051271096 & 1.051271100 & $4 \times 10^{-9}$ \\
0.10 & 1.105170918 & 1.105170960 & $4.2 \times 10^{-8}$ \\
0.15 & 1.161834243 & 1.161834260 & $1.7 \times 10^{-8}$ \\
0.20 & 1.221402758 & 1.221402810 & $5.2 \times 10^{-8}$ \\
0.25 & 1.284025417 & 1.284025390 & $2.7 \times 10^{-8}$ \\
0.30 & 1.349858808 & 1.349858810 & $2 \times 10^{-9}$ \\
0.35 & 1.419067549 & 1.419067540 & $9 \times 10^{-9}$ \\
0.40 & 1.491824698 & 1.491824670 & $2.8 \times 10^{-8}$ \\
0.45 & 1.568312185 & 1.568312140 & $4.5 \times 10^{-8}$ \\
0.50 & 1.648721271 & 1.648721240 & $3.1 \times 10^{-8}$ \\
0.55 & 1.733253018 & 1.733253050 & $3.2 \times 10^{-8}$ \\
0.60 & 1.822118800 & 1.822118800 & 0 \\
0.65 & 1.915540829 & 1.915540860 & $3.1 \times 10^{-8}$ \\
0.70 & 2.013752707 & 2.013752750 & $4.3 \times 10^{-8}$ \\
0.75 & 2.117000017 & 2.116999990 & $2.7 \times 10^{-8}$ \\
\hline
\end{tabular}




\begin{tabular}{cccc}
\hline $\mathrm{t}$ & Exact $\mathrm{s}(\mathrm{t})$ & HAM & Absolute Error \\
\hline 0.80 & 2.225540928 & 2.225540890 & $3.8 \times 10^{-8}$ \\
0.85 & 2.339646852 & 2.339646830 & $2.2 \times 10^{-8}$ \\
0.90 & 2.459603111 & 2.459603100 & $1.1 \times 10^{-8}$ \\
0.95 & 2.585709659 & 2.585709680 & $2.1 \times 10^{-8}$ \\
1.0 & 2.718281828 & 2.718281790 & $3.8 \times 10^{-8}$ \\
\hline
\end{tabular}

Table 3: Results for $d s / d t$

\begin{tabular}{|c|c|c|c|}
\hline $\mathrm{t}$ & Exact $d s / d t$ & HAM & Absolute Error \\
\hline 0.0 & -1 & -1 & 0 \\
\hline 0.05 & -1.051271096 & -1.051271100 & $4 \times 10^{-9}$ \\
\hline 0.10 & -1.105170918 & -1.105170960 & $4.2 \times 10^{-8}$ \\
\hline 0.15 & -1.161834243 & -1.161834260 & $1.7 \times 10^{-8}$ \\
\hline 0.20 & -1.221402758 & -1.221402810 & $5.2 \times 10^{-8}$ \\
\hline 0.25 & -1.284025417 & -1.284025390 & $2.7 \times 10^{-8}$ \\
\hline 0.30 & -1.349858808 & -1.349858810 & $2 \times 10^{-9}$ \\
\hline 0.35 & -1.419067549 & -1.419067540 & $9 \times 10^{-9}$ \\
\hline 0.40 & -1.491824698 & -1.491824670 & $2.8 \times 10^{-8}$ \\
\hline 0.45 & -1.568312185 & -1.568312140 & $4.5 \times 10^{-8}$ \\
\hline 0.50 & -1.648721271 & -1.648721240 & $3.1 \times 10^{-8}$ \\
\hline 0.55 & -1.733253018 & -1.733253050 & $3.2 \times 10^{-8}$ \\
\hline 0.60 & -1.822118800 & -1.822118800 & 0 \\
\hline 0.65 & -1.915540829 & -1.915540860 & $3.1 \times 10^{-8}$ \\
\hline 0.70 & -2.013752707 & -2.013752750 & $4.3 \times 10^{-8}$ \\
\hline 0.75 & -2.117000017 & -2.116999990 & $2.7 \times 10^{-8}$ \\
\hline 0.80 & -2.225540928 & -2.225540890 & $3.8 \times 10^{-8}$ \\
\hline 0.85 & -2.339646852 & -2.339646830 & $2.2 \times 10^{-8}$ \\
\hline 0.90 & -2.459603111 & -2.459603100 & $1.1 \times 10^{-8}$ \\
\hline 0.95 & -2.585709659 & -2.585709680 & $2.1 \times 10^{-8}$ \\
\hline 1.0 & -2.718281828 & -2.718281790 & $3.8 \times 10^{-8}$ \\
\hline
\end{tabular}

\section{Conclusion}

The numerical results obtained by HAM are in good agreement with the exact values for the temperature distribution $\mathrm{u}(\mathrm{x}, \mathrm{t})$, the moving boundary s $(\mathrm{t})$ and the Stefan condition. The freedom in choosing the convergence-control parameter $\mathrm{h}$ enables us to adjust and control the convergence of the solution series and this differentiates the homotopy analysis method from other existing methods such as the homotopy perturbation method, Adomian decomposition method and variational iteration method.

MAPLE was used for the computation presented in this paper.

\section{Acknowledgements}

The author would like to thank the referees for their constructive suggestions and valuable comments.

\section{References}

[1] A.K.Alomari, Modifications of Homotopy Analysis Method for Differential Equations: Modifications of Homotopy Analysis Method, Ordinary, Fractional, Delay and Algebraic Equations, Lambert Academic Publishing, Germany, 2012.

[2] A.Fasano, M. Primicerio,Free Boundary problems for nonlinear parabolic equations with nonlinear free boundary conditions, J.Math.Anal.Appl,72(1979),247 -273. 
[3] D. Slota, Homotopy Perturbation Method for solving the two-phase inverse Stefan problem, Numer. Heat Trans, 59(2011), 755-768.

[4] H. Jafari, M.Saeidy and M.A.Frozjaei, Homotopy Analysis Method: a tool for solving a Stefan Problem, J. Adv Res. Sci. Comput, 2(2010), 61-68.

[5] H.Jafari,A.Golbabai,E.Salehpoor and Kh. Sayehvand, Application of Variational Iteration Method, Appl.Math.Sci, $2(2008), 3001$-3004.

[6] O.O.Onyejekwe and O.N.Onyejekwe, Numerical solutions of the one-phase classical Stefan Problem using an enthalpy green element method, Adv. Eng. Soft, 42(2011), $743-749$.

[7] R. Grzymkowski, M.Pleszczyński and D.Slota, Comparing the Adomian Decomposition Method and the Runge-Kutta Method for solutions of the Stefan problem, Intl.J. Comput.83 (2006), $409-417$.

[8] R. Grzymkowski and D.Slota, Stefan Problem solved by Adomian Decomposition Method, Intl. J. of Comput. Math, 82(2005), $851-856$.

[9] S. Kutluay, Numerical Schemes for one-dimensional Stefan - like problems with a forcing term, Appl. Math. And Comput, 168(2005), 11591168 .

[10] S. Kutluay, A.R.Bahadir and A.Ozdes, The numerical solution of one-phase classical Stefan problem, J. Comput. And Appl. Math, 81(1997), 135-144.

[11] S.Liao, Homotopy Analysis Method in Nonlinear Equations, Springer, New York, 2012.

[12] S.Liao, Beyond Perturbation: Introduction to homotopy Analysis Method, Chapman \& Hall CRC, 2004.

[13] S.Liao, Notes on the homotopy analysis method- Some definitions and theorems, Common, Nonlinear Sci. Numer. Simulat, 14(2009), 983997.

[14] S.L.Mitchell and T.G.Myers, Application of Heat Balance Integral Methods to one-dimensional phase change problems, Intl. J. Diff. Equa, 2012, 1-22.

[15] S. Savovic and J.Caldwell, Numerical Solution of Stefan Problem with time-dependent boundary conditions by variable space grid method, Ther. Sci, 13(2009) 165-174.

[16] S. Savovic and J.Caldwell, Finite Difference solution of one-dimensional Stefan problem with periodic boundary conditions, Intl.J. Heat. Mass Transr, 46 (2003), 2911-2916.

[17] T.C.Smith, A finite difference method for a Stefan problem, CALCOLO, 18(1981), 131-154. 